\title{
A Study of $f_{0}(1500)$ decays into $4 \pi^{0}$ in $\bar{p} p \rightarrow 5 \pi^{0}$ at Rest
}

\section{Crystal Barrel Collaboration}

A. Abele ${ }^{h}$, J. Adomeit ${ }^{g}$, C. Amsler ${ }^{p}$, D.S. Armstrong ${ }^{a 1}$, C.A. Baker ${ }^{e}$, B.M. Barnett ${ }^{c}$, C.J. Batty ${ }^{e}$, M. Benayoun ${ }^{m}$, A. Berdoz ${ }^{l}$ K. Beuchert ${ }^{b}$, P. Birien ${ }^{a}$, S. Bischoff ${ }^{h}$, P. Blüm ${ }^{h}$, K. Braune ${ }^{n}$, J. Brose ${ }^{k}$, D.V. Bugg ${ }^{i}$, T. Case ${ }^{a}$, A. Cooper ${ }^{i}$, O. Cramer ${ }^{n}$, K.M. Crowe ${ }^{a}$, T. Degener ${ }^{b}$, H.P. Dietz ${ }^{n}$, N. Djaoshvili ${ }^{n}$, S. v. Dombrowski ${ }^{p}$, M. Doser ${ }^{f}$, W. Dünnweber ${ }^{n}$, D. Engelhardt ${ }^{h}$, M. Englert ${ }^{n}$, M.A. Faessler ${ }^{n}$, P. Giarritta ${ }^{p}$, R. Hackmann ${ }^{c}$, R.P. Haddock ${ }^{j}$, F.H. Heinsius ${ }^{a}$, M. Herz ${ }^{c}$,

N.P. Hessey ${ }^{f}$, P. Hidas ${ }^{d}$, C. Holzhaussen ${ }^{h}$, P. Illinger ${ }^{n}$, D. Jamnik ${ }^{2}$, H. Kalinowsky ${ }^{c}$, B. Kalteyer ${ }^{c}$, B. Kämmle ${ }^{g}$, P. Kammel ${ }^{a}$, T. Kiel ${ }^{h}$, J. Kisiel ${ }^{f 3}$, E. Klempt ${ }^{c}$, H. Koch ${ }^{b}$, C. Kolo $^{n}$, M. Kunze ${ }^{b}$, M. Lakata ${ }^{a}$, R. Landua ${ }^{f}$, J. Lüdemann ${ }^{b}$, H. Matthaey ${ }^{b}$, R. McCrady ${ }^{l}$, J. Meier ${ }^{g}$, J.P. Merlo ${ }^{k}$, C.A. Meyer ${ }^{l}$, L. Montanet ${ }^{f}$, A. Noble ${ }^{p}$, R. Ouared ${ }^{f}$, F. Ould-Saada ${ }^{p}$, K. Peters ${ }^{b}$, C.N. Pinder ${ }^{e}$, G. Pinter ${ }^{d}$, S. Ravndal ${ }^{b 5}$, C. Regenfus ${ }^{n}$, J. Reißmann ${ }^{g}$, S. Resag ${ }^{c 6}$, W. Röthel ${ }^{n}$, E. Schäfer ${ }^{k}$,

P. Schmidt ${ }^{g}$, I. Scott ${ }^{i 6}$, R. Seibert ${ }^{g}$, S. Spanier ${ }^{p}$, H. Stöck ${ }^{b}$, C. Straßburger ${ }^{c}$, U. Strohbusch ${ }^{g}$, M. Suffert ${ }^{\circ}$, U. Thoma ${ }^{c}$, M. Tischhäuser ${ }^{h}$, D. Urner ${ }^{p}$, C. Völcker ${ }^{n}$, F. Walter ${ }^{k}$, D. Walther ${ }^{b}$ U. Wiedner ${ }^{g}$, J. Zoll ${ }^{f}$, B.S. Zou ${ }^{i}$, Č. Zupančič ${ }^{n}$

a University of California, LBL, Berkeley, CA 94720, USA

${ }^{b}$ Universität Bochum, D-44780 Bochum, FRG

${ }^{c}$ Universität Bonn, D-53115 Bonn, FRG

d Academy of Science, H-1525 Budapest, Hungary

e Rutherford Appleton Laboratory, Chilton, Didcot OX11 OQX, UK

f CERN, CH-1211 Genève, Switzerland

9 Universität Hamburg, D-22761 Hamburg, FRG

${ }^{h}$ Universität Karlsruhe, D-76021 Karlsruhe, FRG

i Queen Mary and Westfield College, London E1 4NS, UK

3 University of California, Los Angeles, CA 90024, USA

${ }^{k}$ Universität Mainz, D-55099 Mainz, FRG

1 Carnegie Mellon University, Pittsburgh, PA 15219, USA

${ }^{m}$ LPNHE Paris VI, VII, F-75252 Paris, France

${ }^{n}$ Universität München, D-80393 München, FRG

- Centre de Recherches Nucléaires, F-67037 Strasbourg, France

$p$ Universität Zürich, CH-8057 Zürich, Switzerland

Received ; revised manuscript received USA

${ }^{1}$ William \& Mary College, Williamsburg, VA 23187,

${ }^{2}$ University of Ljubljana, Ljubljana, Slovenia

${ }^{3}$ University of Silesia, Katowice, Poland

${ }^{4}$ Now at CRPP, Ottawa, Canada

${ }^{5}$ Now at CERN, Genève, Switzerland

${ }^{6}$ This work is part of the PhD theses of S. Resag and I.
Crystal Barrel data on $\bar{p} p \rightarrow 5 \pi^{0}$ at rest are presented. Mass spectra for $2 \pi^{0}$ combinations, $3 \pi^{0}$ and $4 \pi^{0}$ and decay angular distributions all differ significantly from phase space. The $2 \pi^{0}$ mass spectrum agrees closely with CERNMunich results. We present several ways of fitting the data. All agree on the definite presence of the $f_{0}(1500)$, observed in its $4 \pi^{0}$ decay mode. It can decay via a $\pi \pi^{*}$ or a $\sigma \sigma$ inter- 
mediate state, where $\pi^{*}$ represents the heavy pion called $\pi(1300)$ by the Particle Data Group, and $\sigma$ stands for the full $\pi \pi$ S-wave amplitude.

In earlier publications, we have presented evidence for a new $I=0, J^{P C}=0^{++}$resonance $f_{0}(1500)$ decaying to $\pi^{0} \pi^{0}, \eta \eta$ and $\eta \eta^{\prime}[1]-[6]$. The $\mathrm{N} / \mathrm{D}$ method, used in [2], was described fully in [7]-[9], which give further results. In [3]-[5] a Pvector approach was adopted; this method is described in detail in [10]. A second isoscalar scalar resonance at a mass of $1300-1370 \mathrm{MeV}$, decaying into $\pi \pi$ and $\eta \eta$, is definitely required by the data, though its width is less certain. In [7] and [9] its width is comparatively narrow $(\approx 250 \mathrm{MeV})$, while in analyses based on the $\mathrm{P}$ vector approach, it may also be broad $(\approx 700 \mathrm{MeV})$ and could be describing t-channel exchanges [11] [12]. There is evidence in $J / \Psi$ radiative decays for further resonances $f_{0}(1750)$ and $f_{0}(2104)$ [13].

Earliest evidence for a $I=0, J^{P}=0^{+}$resonance at $(1300-1400) \mathrm{MeV}$ came from $4 \pi$ data of Bettini et al. [14], who proposed a resonance decaying to $\rho \rho$. Later analyses claimed that this reaction is dominated by one tensor state with a mass of $1480 \mathrm{MeV}$ [15]. This was challenged in a reanalysis by Gaspero, who found scalar quantum numbers and $\mathrm{M}=(1386 \pm 38) \mathrm{MeV}, \Gamma=(310 \pm 64)$ $\mathrm{MeV}[16]$. The OBELIX group presented similar evidence for a $0^{+}$resonance at $\mathrm{M}=(1345 \pm 12)$ $\mathrm{MeV}, \Gamma=(398 \pm 26) \mathrm{MeV}$, decaying $29 \%$ to $\rho \rho$ and $71 \%$ to $\sigma \sigma$ in $\pi^{+} \pi^{-} \pi^{+} \pi^{-}$[17]. We ourselves observed a similar resonance at $(1374 \pm 38) \mathrm{MeV}$, with width $(375 \pm 61) \mathrm{MeV}$, but decaying more strongly to $\rho \rho(61 \%)$ than $\sigma \sigma(39 \%)$ [18]. Here we examine $\bar{p} p \rightarrow 5 \pi^{0}$ for the light it sheds on $\sigma \sigma$ decay modes, since $\rho \rightarrow \pi^{0} \pi^{0}$ is forbidden.

The data have been collected with the Crystal Barrel detector at LEAR. This detector is well suited to study the $5 \pi^{0}$ channel, because of its excellent $\gamma$ detection. Since it has been described in detail elsewhere [19], only a short summary will be given here.

A $200 \mathrm{MeV} / \mathrm{c} \bar{p}$ beam stops in a liquid hydrogen target at the center of the detector. The target is surrounded by a pair of cylindrical multiwire proportional chambers (PWC's) and a cylindrical drift chamber (JDC). The JDC is surrounded by $1380 \mathrm{CsI}(\mathrm{Tl})$ crystals, pointing towards the target center. Typical photon resolutions are $\sigma_{E} / E=$ $2.5 \%$ at $1 \mathrm{GeV}$, and $\sigma=1.2^{\circ}$ in both polar and azimuthal angles. The calorimeter is fully efficient over $95 \%$ of $4 \pi$, extending in polar angle from $12^{\circ}$ to $168^{\circ}$; extra photons are vetoed over $98 \%$ of $4 \pi$.

The data for the present analysis are taken with a zero-prong trigger requiring the absence of any charged particle in either PWC. They are required to satisfy the following criteria:

- No charged tracks in the JDC

- Exactly 10 photons with energy above $20 \mathrm{MeV}$

- Energy of the central crystal $>10 \mathrm{MeV}$, to avoid split-offs (which mostly have low energies)

- No photons should have maximum energy deposit in a crystal adjoining the beam pipe (to avoid shower leakage)

- Energy and momentum conservation:

$1.6 \mathrm{GeV} \leq \mathrm{E}_{\text {tot }} \leq 2.1 \mathrm{GeV},\left|\mathbf{P}_{\text {tot }}\right| \leq 0.16 \mathrm{GeV}$.

Data surviving these cuts undergo kinematic fitting to the $\bar{p} p \rightarrow 10 \gamma$ final state (4C fit) and in a second step to $\bar{p} p \rightarrow 5 \pi^{0}$ and $\bar{p} p \rightarrow 4 \pi^{0} \eta$ (9C). In order to separate the $5 \pi^{0}$ and $4 \pi^{0} \eta$ final states, events satisfying both hypotheses are assigned to whichever channel gives the better confidence level, weighted according to production branching ratios. Events are rejected if the confidence level is below $10 \%$ or if there is more than one good fit to the $5 \pi^{0}$ hypothesis.

The $5 \pi^{0}$ sample contains a negligible contamination $(<1 \%)$ of non $5 \pi^{0}$ annihilations. A combinatorial background due to wrong pairing of the $10 \gamma$ is estimated, by Monte Carlo, to be $3 \%$ of the $5 \pi^{0}$ sample.

Amongst the accepted $5 \pi^{0}$ events, there is a clear signal due to $\eta \pi^{0} \pi^{0}$, followed by $\eta \rightarrow 3 \pi^{0}$. This signal is rejected by eliminating all events with $523 \mathrm{MeV}<m_{3 \pi^{0}}<575 \mathrm{MeV}$. Further details of data-processing and event selection are given in full in two theses [20] [21].

Ultimately, 25000 data-events are used for analysis, together with 45000 Monte Carlo events satisfying identical kinematic selection. Using the Monte Carlo simulation to derive the detection efficiency, we find that the branching ratio for $\bar{p} p$ annihilation at rest to $5 \pi^{0}$ (excluding $\eta \pi^{0} \pi^{0}$ ) is 

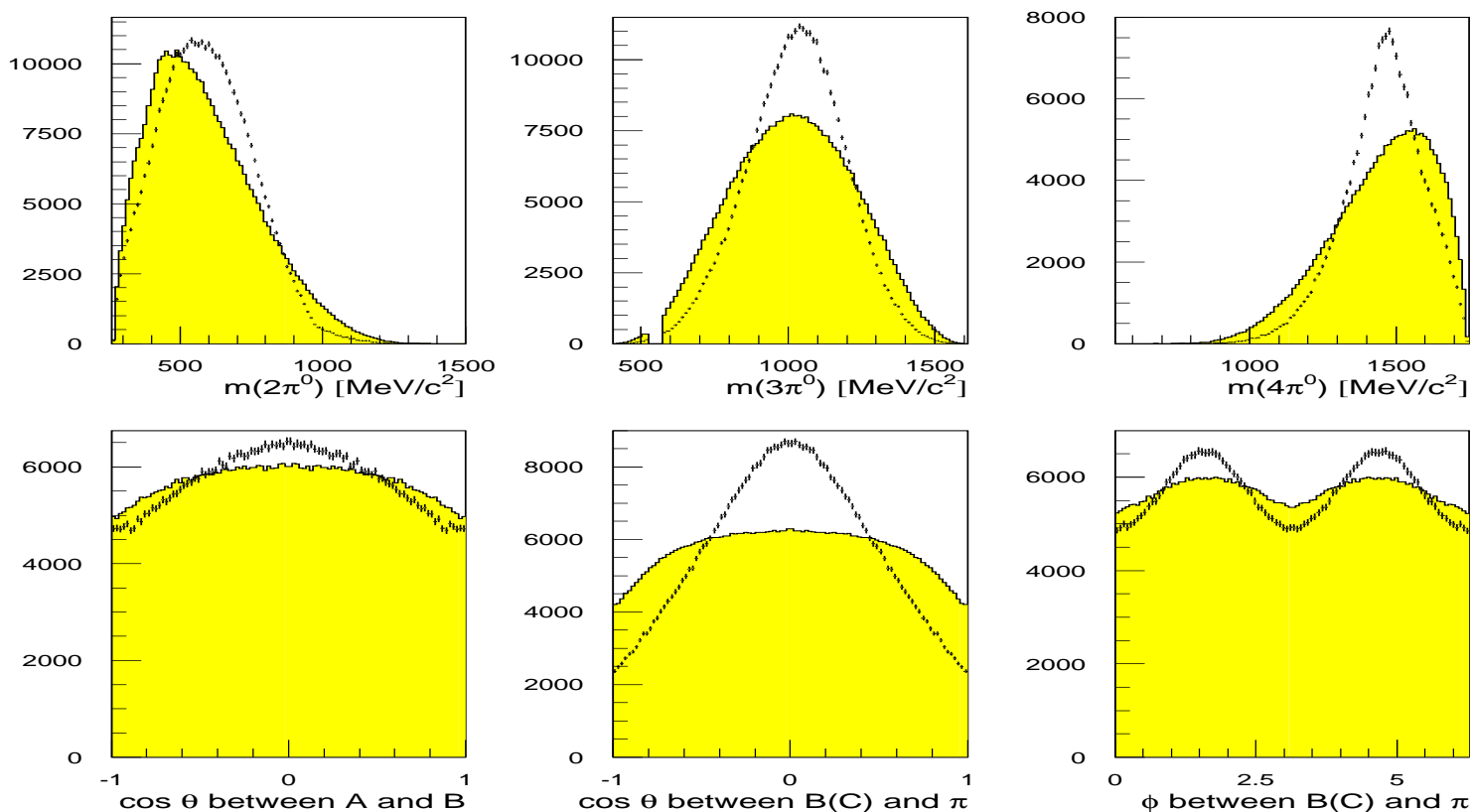

Fig. 1. Invariant mass and decay angular distributions of data (shown as points with error-bars) compared to MC phase space (shaded). For a definition of the angles, see Fig. 2

$0.71 \%$, with a systematic error of $\pm 0.10 \%$. This branching ratio has been checked against $\bar{p} p \rightarrow$ $\eta \pi^{0} \pi^{0}$, followed by $\eta \rightarrow 3 \pi^{0}$. We find $\mathrm{B}(\bar{p} p \rightarrow$ $\pi \pi \eta)=(8.2 \pm 1.0) \cdot 10^{-3}$, in good agreement with our previous determination $(6.7 \pm 1.2) \cdot 10^{-3}$ using six photons as final state [6]. The Dalitz plot for $\eta \pi^{0} \pi^{0}$ from the $10 \gamma$ final states has been checked against that from $6 \gamma$ events, and is in excellent agreement [20].

Mass spectra for $2 \pi^{0}, 3 \pi^{0}$ and $4 \pi^{0}$ combinations and three decay angular distributions are shown in Fig. 1. All show significant deviations from phase space (shaded). Note that the angular distributions for phase space depart from isotropy because of acceptance (mostly due to the rejection of events with more than one good fit to $5 \pi^{0}$ and the $E_{\gamma}>20 \mathrm{MeV}$ cut). We get nearly flat angular distributions if we accept events with $\geq$ one good $5 \pi^{0}$ fit, but the combinatorical background then increases to $(11-12) \%$.

We consider first the $2 \pi$ mass distribution, Fig. 1(a). Compared with phase space, it is biased towards high masses by the $\pi \pi$ S-wave interaction, which reaches a phase shift of $90^{\circ}$ around 800 $\mathrm{MeV}$. The $\pi \pi \mathrm{S}$-wave has been fitted to $\pi \pi$ data [22] [23] and to the $\bar{p} p \rightarrow 3 \pi^{0}$ data of [4]. It reproduces the $2 \pi$ mass distribution accurately in all fits. We remark that Svec has proposed a narrow $f_{0}(750)$ resonance with a full width of $(200-300)$ $\mathrm{MeV}$ [24]. This fails to fit our data. Using it with a width of $250 \mathrm{MeV}$, we have carried out full fits, and a typical fit to the $2 \pi$ mass spectrum is shown in Fig. 3.

Next we come to the $3 \pi$ mass spectrum. It peaks around $1050 \mathrm{MeV}$. This cannot, however, immediately be interpreted as a resonance because of the large combinatorical background (10 entries per event). Finally, in the $4 \pi$ mass spectrum, 5 entries per event, we observe a peak at about 1450 $\mathrm{MeV}$, which suggests the presence of resonances decaying into $4 \pi^{0}$.

The C-parity of $5 \pi^{0}$ is +1 . Hence S-state annihilation is possible only from ${ }^{1} S_{0}$. In view of the complexity of $5 \pi$ final states, and the dominance of S-state annihilation in liquid, we ignore P-state annihilation. We drop $f_{2}(1270)$ and $a_{1}(1260)$ from 


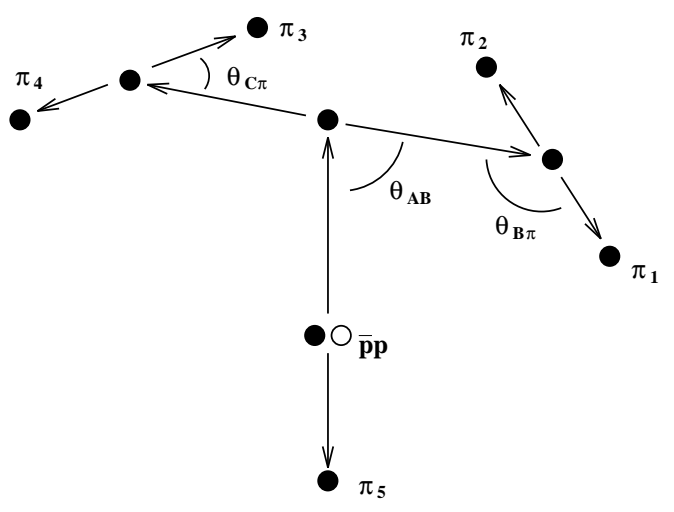

Fig. 2. Definition of the decay angles. All angles are defined in the rest frame of the decaying particle.

the further analysis for the following reasons. The branching ratio for $\bar{p} p \rightarrow f_{2}(1270) \pi \rightarrow 5 \pi^{0}$ can be calculated from the $\bar{p} p \rightarrow f_{2}(1270) \pi^{0}$ channel in $3 \pi^{0}$ data [6], and the branching ratios of $f_{2}(1270)$ to $\pi^{0} \pi^{0}$ and $4 \pi^{0}$ [25]. The predicted $f_{2}(1270) \rightarrow 4 \pi^{0}$ signal is $0.09 \%$ of all $5 \pi^{0}$ data. Fitting this channel improves log likelihood by only 8 , a negligible amount. The $a_{1} \rightarrow \sigma \pi$ branching ratio quoted by the $\mathrm{PDG}$ is $<0.7 \%$. Again, it gives negligible improvement in the fit.

We shall fit the following channels to the data, using the maximum likelihood method:

$$
\begin{aligned}
\bar{p} p & \rightarrow f_{0} \pi \rightarrow(\sigma \sigma) \pi \\
& \rightarrow f_{0} \pi \rightarrow\left(\pi \pi^{*}\right) \pi \rightarrow([\sigma \pi] \pi) \pi \\
& \rightarrow \pi^{*} \sigma \rightarrow(\sigma \pi) \sigma .
\end{aligned}
$$

We minimize $S$ defined by

$$
S=-2 \ln L=2 N \ln \left(\sum_{i=1}^{M} w_{i}\right)-2\left(\sum_{j=1}^{N} \ln w_{j}\right)
$$

Here $N$ is the number of data events, $M$ the number of Monte Carlo events and $w$ is the weight for the kinematics of a particular event.

The partial wave analysis assumes the isobar model. Amplitudes $A$ are constructed as

$A=F \cdot T_{1} \cdot T_{2}+$ permutations.

For the process $\bar{p} p \rightarrow f_{0} \pi^{0}, F$ is given by a BreitWigner formula with constant width:

$F_{j, \alpha}=\frac{a_{j, \alpha} \cdot e^{i \phi_{j, \alpha}}}{m_{\alpha}^{2}-m^{2}-i m_{\alpha} \Gamma_{\alpha}^{0}}$.

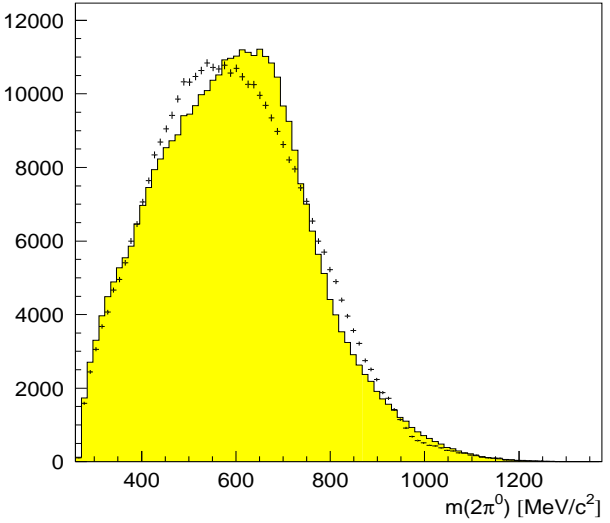

Fig. 3. $2 \pi^{0}$ invariant mass distribution, using an $f_{0}(750)$ resonance with a width of $250 \mathrm{MeV}$ for description of the $\pi \pi$ S-wave (shaded), compared with data.

The index $j$ denotes the two decay chains of (1) and $\alpha$ specifies the $4 \pi^{0}$ resonance. We have tried including mass-dependence into the full width. Although numerical values of terms in (3) change, the quality of the fit alters little, and pole positions of resonances are stable. For a full discussion of this issue in a slightly different context, see [26].

$T_{1}$ and $T_{2}$ describe the $\pi \pi$-scattering amplitudes evaluated at the masses of the $2 \pi^{0}$ subsystems. Several prescriptions have been tried, notably those from [4] and [12]. The dependence of the result on the prescription is not significant, provided its parameters fit CERN-Munich data [23]. For the process $\bar{p} p \rightarrow f_{0} \pi^{0} \rightarrow \pi^{* 0} \pi^{0} \pi^{0} \rightarrow$ $\sigma \pi^{0} \pi^{0} \pi^{0}, T_{1}$ is a relativistic Breit-Wigner describing the $\pi^{*}$.

Since we have five identical particles in the final state, the decay chain $\bar{p} p \rightarrow f_{0} \pi \rightarrow(\sigma \sigma) \pi$ is summed coherently over 15 configurations, and the chain $\bar{p} p \rightarrow f_{0} \pi \rightarrow\left(\pi \pi^{*}\right) \pi \rightarrow([\sigma \pi] \pi) \pi$ over 60 configurations. In all fits we use an incoherent phase-space background of $4 \%$ to describe wrongly paired photons and background from other channels.

We now discuss fits to the data. The simplest, entry 1 of Tab. 1, uses a single scalar resonance decaying to $\sigma \sigma$. The mass and width found for the resonance are consistent with previous work [16] - [18]. However, the $4 \pi^{0}$ mass distribution is not described accurately, see Fig. 4. The description 


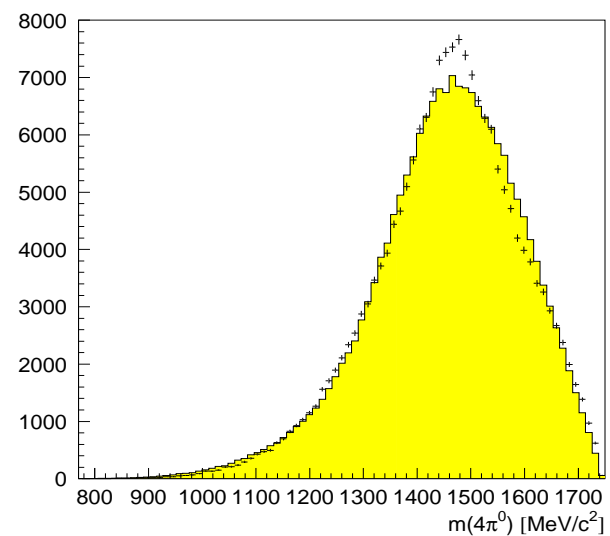

Fig. 4. $4 \pi^{0}$ invariant mass distribution. Comparison of fit 1 and data, using one scalar with $\mathrm{M}=1395 \mathrm{MeV}$ and $\Gamma=364$ $\mathrm{MeV}$.

may be improved significantly when more resonances are introduced.

Since the $4 \pi$ mass spectrum peaks below $f_{0}(1500)$, we first try a fit with $f_{0}(1500)$ and a second $f_{0}$ resonance in the mass range $(1300-1370)$ $\mathrm{MeV}$ and with a width of $(230-380) \mathrm{MeV}$. This fit is no better than fit 1 in Fig. 4, so it is not shown in Table 1. Next, a close inspection of the $4 \pi$ mass spectrum shows a slight shoulder above $1600 \mathrm{MeV}$. We find that satisfactory fits can be obtained by adding $f_{0}(1750)$ to $f_{0}(1300)$ and $f_{0}(1500)$. This resonance is at the limit of phase space and present data do not determine its mass and width, which we take from [13]. Entry 2 of Table 1 shows this solution.

Below, we shall elaborate on the requirement for $f_{0}(1500) \rightarrow \pi \pi^{*}$. Entry 3 of Table 1 shows the substantial improvement (491) in log likelihood obtained by adding this decay, but keeping the masses and widths of $f_{0}(1300)$ and $\pi^{*}$ fixed. For the latter, the mass and width are a compromise amongst previous publications quoted by the PDG. Entry 4 shows the modest improvement obtained by adding further channels $\bar{p} p \rightarrow \sigma \pi^{*}$ and $f_{0}(1750) \rightarrow \pi \pi^{*}$ (4 extra parameters in the fit).

The masses and widths of $f_{0}(1300), f_{0}(1750)$ are not well determined from $5 \pi^{0}$ data. In addition to $f_{0}(1500)$, some component giving a broad $4 \pi$ mass spectrum is required. Entries 5 to 8 of Table 1 show fits obtained by relaxing progres-
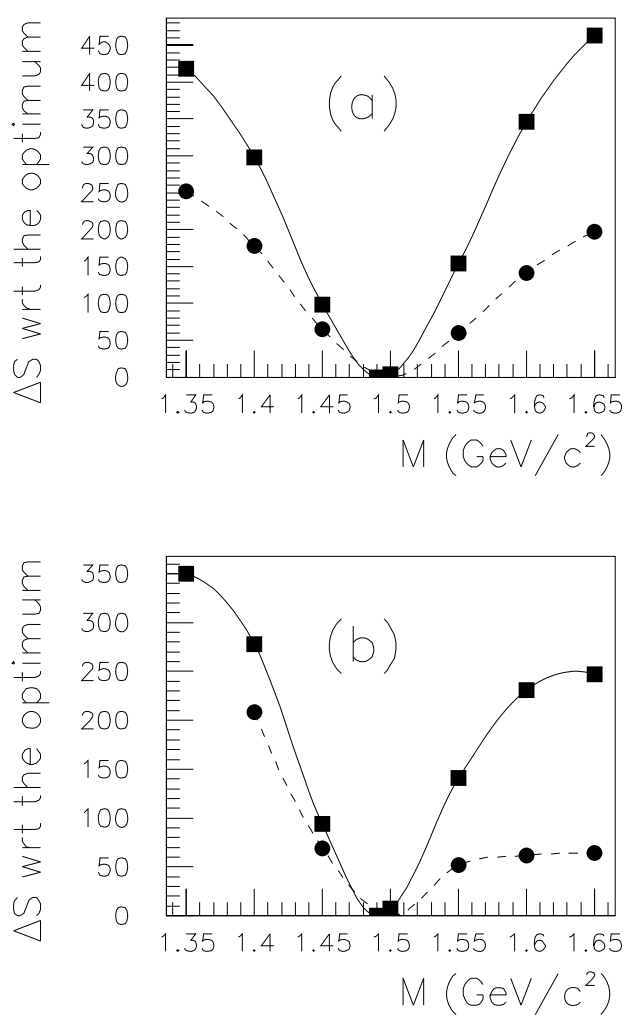

Fig. 5. $-2 \ln L$ v. mass fitted to $f_{0}(1500)$, (a) for $\sigma \sigma$ decays, (b) for $\pi \pi^{*}$ decays. Full curves are obtained with the components of fit 3 and dashed curves with the components of fit 7 . Both are normalised to 0 at their minima.

sively the constraints on the masses and widths of $\pi^{*}$ and $f_{0}(1300)$. A free fit of $\pi^{*}$ to present data gives $M=1114 \mathrm{MeV}, \Gamma=340 \mathrm{MeV}$. The fit is also improved by allowing the width of $f_{0}(1300)$ to increase to a very large value $(\sim 1000) \mathrm{MeV}$. Entry 5 is a compromise between these alternatives, freeing only $\pi^{*}$.

We now come to our central point concerning $f_{0}(1500)$. Definite evidence for two decay modes $\sigma \sigma$ and $\pi \pi^{*}-$ is found. Its decay to $\sigma \sigma$ has already been reported in $J / \Psi$ radiative decays [13]. We find it to be essential here. In addition, we find that, in all fits, the inclusion of the decay mode to $\pi \pi^{*}$ improves $2 \ln L$ by $\sim 500$, a highly significant amount. For one degree of freedom, a change of $2 \cdot \ln L$ of 1 represents one standard deviation. 


\begin{tabular}{|c|c|c|c|c|c|c|c|c|c|c|c|c|c|}
\hline & \multicolumn{3}{|c|}{$\sigma \pi^{*}$} & \multicolumn{3}{|c|}{$f_{0}(1300) \pi$} & \multicolumn{4}{|c|}{$f_{0}(1500) \pi$} & \multicolumn{2}{|c|}{$f_{0}(1750) \pi$} & \multirow[t]{2}{*}{$-2 \cdot \ln L$} \\
\hline & $\mathrm{m}$ & $\Gamma$ & $\sigma \pi$ & $\mathrm{m}$ & $\Gamma$ & $\sigma \sigma$ & $\mathrm{m}$ & $\Gamma$ & $\sigma \sigma$ & $\pi \pi^{*}$ & $\sigma \sigma$ & $\pi \pi^{*}$ & \\
\hline 1 & & - & & 1395 & 364 & $96 \%$ & \multicolumn{4}{|c|}{-} & \multicolumn{2}{|c|}{-} & 1277 \\
\hline 2 & & - & & $1300^{\dagger}$ & $230^{\dagger}$ & $18 \%$ & $1500^{\dagger}$ & $150^{\dagger}$ & $46 \%$ & - & $32 \%$ & - & 677 \\
\hline 3 & $1260^{\dagger}$ & $250^{\dagger}$ & - & $1300^{\dagger}$ & $230^{\dagger}$ & $11 \%$ & $1500^{\dagger}$ & $150^{\dagger}$ & $51 \%$ & $15 \%$ & $19 \%$ & - & 186 \\
\hline 4 & $1260^{\dagger}$ & $250^{\dagger}$ & $4 \%$ & $1300^{\dagger}$ & $230^{\dagger}$ & $1 \%$ & $1500^{\dagger}$ & $150^{\dagger}$ & $45 \%$ & $13 \%$ & $13 \%$ & $20 \%$ & 87 \\
\hline 5 & 1100 & 348 & $12 \%$ & $1300^{\dagger}$ & $230^{\dagger}$ & $2 \%$ & $1500^{\dagger}$ & $150^{\dagger}$ & $21 \%$ & $8 \%$ & $32 \%$ & $20 \%$ & 40 \\
\hline 6 & 1093 & 330 & $6 \%$ & 1065 & 1079 & $67 \%$ & $1500^{\dagger}$ & $150^{\dagger}$ & $8 \%$ & $15 \%$ & - & - & 64 \\
\hline 7 & 1111 & 340 & $10 \%$ & 1198 & 991 & $65 \%$ & $1500^{\dagger}$ & $150^{\dagger}$ & $8 \%$ & $10 \%$ & $0 \%$ & $4 \%$ & 3 \\
\hline 8 & 1114 & 340 & $8 \%$ & 1250 & 1168 & $64 \%$ & 1505 & 169 & $12 \%$ & $9 \%$ & $0 \%$ & $3 \%$ & 0 \\
\hline
\end{tabular}

Fit-results. The numbers in $\sigma \pi, \sigma \sigma$ and $\pi \pi^{*}$ columns give the contributions of these decay modes to the $5 \pi^{0}$ final state (without $\bar{p} p \rightarrow \eta \pi \pi$ ). '-' means that this contribution is not fitted; $0 \%$ means this contribution is allowed, but rejected by the fit. A ' $\dagger$ ' means that the parameter is fixed. Note, that the contributions add up to $96 \%$ due to a $4 \%$ background contribution. $-2 \cdot \ln L$ is normalised to the best fit, entry 8 .
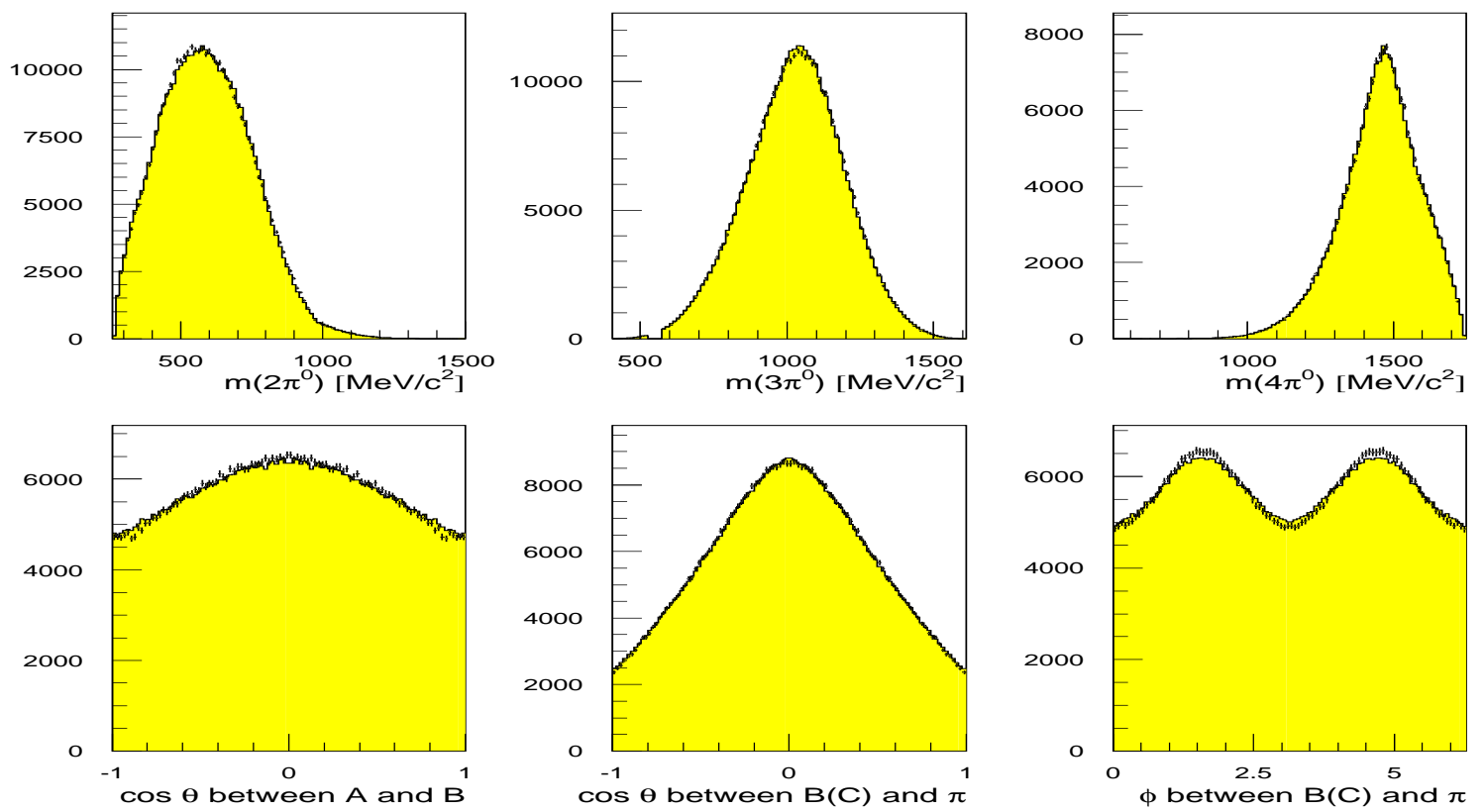

Fig. 6. Invariant mass and decay angular distributions of data (shown as points with error-bars) compared to fit 7 (shaded). 
In order to demonstrate the presence of both decay modes, we scan the mass of the $f_{0}$ resonance. This is done separately for the two decays modes. In Fig. 5(a), the mass and width for the $\pi \pi^{*}$ decay mode are held fixed, while the mass for the $\sigma \sigma$ decay mode is varied, keeping the width fixed at $150 \mathrm{MeV}$. In Fig. 5(b), the reverse is shown: the mass and width of the $\sigma \sigma$ decay mode are kept fixed, while the mass for the $\pi \pi^{*}$ decay mode is varied, again keeping the width fixed at $150 \mathrm{MeV}$. Both scans give well determined minima at 1500 $\mathrm{MeV}$. This is so, regardless of whether we use mass and width for the $f_{0}(1300)$ and $\pi^{*}$ from fit 3 (full curves) or from fit 7 (dashed curves). When they are taken from fit 7 , the minima are of course shallower, because of larger interference possibilities; but they are still significant. If both decays are scanned together with a single mass and width, the optimum is even deeper and sharper. Individual projections of Fig. 1 do not distinguish between $\sigma \sigma$ and $\pi \pi^{*}$ decay modes. The maximum likelihood fit, which includes all combinatorics, is what is sensitive to the presence of both of them. The quality of the fit to projections is shown in Fig. 6. It is indistinguishable by eye between fits $3-8$.

The contribution of each channel to the $5 \pi^{0} \mathrm{fi}-$ nal state are given in Tab. 1. However, one cannot immediately deduce branching ratios from these numbers. There is an important subtlety in determining branching ratios for resonance decays. In Tab. 1, there are strong interferences between the resonance and the spectator. As an example, $f_{0}(1500)$ may be formed between five combinations of pions numbered 1234, 1235, 1245, 1345 or 2345. These all interfere. For a resonance decaying freely, i.e. away from the spectator pion, the interferences with the spectator are absent. Taking as an example an $f_{0}$ resonance between particles 1234 decaying only to $\sigma \sigma$ for simplicity, the required cross-section $\mathrm{d} \sigma_{1234} / \mathrm{d} \Omega$ is

$\frac{d \sigma_{1234}}{d \Omega}=\left|T_{12} T_{34}+T_{13} T_{24}+T_{14} T_{23}\right|^{2}|F|^{2}$,

where $T_{12}$ is the $\sigma$ amplitude for particles 1 and 2 and $F$ is the Breit-Wigner amplitude for the resonance in question. This expression includes all interferences between these four particles but ex-

\begin{tabular}{|c||ccc|}
\hline fit & $\begin{array}{c}f_{0}(1500) \\
\rightarrow \sigma \sigma\end{array}$ & $\begin{array}{c}f_{0}(1500) \\
\rightarrow \pi \pi^{*}\end{array}$ & $\begin{array}{c}f_{0}(1500) \\
\rightarrow 4 \pi^{0}\end{array}$ \\
\hline 3 & $19.0 \%$ & $6.3 \%$ & $13.6 \%$ \\
4 & $21.8 \%$ & $7.4 \%$ & $12.8 \%$ \\
5 & $8.1 \%$ & $9.5 \%$ & $14.2 \%$ \\
6 & $2.5 \%$ & $5.5 \%$ & $13.9 \%$ \\
7 & $2.2 \%$ & $3.3 \%$ & $10.1 \%$ \\
8 & $3.5 \%$ & $2.9 \%$ & $11.7 \%$ \\
\hline & & & $(12.7 \pm 1.6) \%$ \\
\hline
\end{tabular}

Branching ratios for $f_{0}(1500)$, compared to $\bar{p} p \rightarrow 5 \pi^{0}$, where interferences with the spectator are ignored.

cludes interferences with particle 5 . We have likewise included interference with the decays $f_{0} \rightarrow$ $\pi \pi^{*}$ amongst particles $1-4$. For the five resonances possible in the final state, the cross section is $5 \times \mathrm{d} \sigma_{1234} / \mathrm{d} \Omega$. In $\bar{p} p \rightarrow 3 \pi^{0}$, the corresponding cross section is $3 \times \mathrm{d} \sigma_{12} / \mathrm{d} \Omega$, where the latter quantity describes the resonance decaying into $\pi^{0} \pi^{0}$ via particles 1 and 2 . This approach to computing branching ratios is different to that used in our earlier publications, refs. [1] - [8].

With the new arithmetic, the branching ratios for $f_{0}(1500)$ are given in Tab. 2 for several fits. Individual channels show quite a large scatter. There are strong interferences between $\sigma \sigma$ and $\pi \pi^{*}$ and the fit is able to shift amplitudes between these decay modes to some extent. The sum including these interferences, shown in the final column of Tab. 2, is more stable: $(0.090 \pm 0.014) \times 10^{-2}$. The branching ratio for all charge states from $4 \pi$ is a factor 9 larger. For $f_{0}(1500) \rightarrow \pi \pi$, the corresponding figure [6] from our $3 \pi^{0}$ data is $(0.238 \pm 0.038) \times 10^{-2}$. Hence

$r=\frac{\mathrm{B}\left(f_{0}(1500) \rightarrow 4 \pi\right)}{\mathrm{B}\left(f_{0}(1500) \rightarrow \pi \pi\right)}=3.4 \pm 0.8 ;$

here $\mathrm{B}\left(f_{0}(1500) \rightarrow 4 \pi\right)$ means $4 \pi$-decays not proceeding via $\rho \rho$.

In conclusion, we have analysed the reaction $\bar{p} p \rightarrow 5 \pi^{0}$ at rest and found that the data are dominated by $4 \pi^{0}$ scalar resonances. This scalar $4 \pi^{0}$ intensity has a more complex structure than proposed in earlier analyses [16] - [18]. We find two alternative ways of fitting the data, one using a very broad scalar resonance, which could represent t-channel exchanges. The other alternative uses $f_{0}(1300)$ with $\mathrm{M}=1300 \mathrm{MeV}, \Gamma=230 \mathrm{MeV}$, from [26]. The existence of a narrow $f_{0}(1300)$ cannot 
be extracted reliably from present data; a reanalysis of the $\pi^{+} \pi^{-} 3 \pi^{0}$ data is in progress. The most distinctive feature is the presence of $f_{0}(1500)$. It decays to $\sigma \sigma$ and $\pi \pi^{*}$ with similar intensities, but the two decay modes interfere and fractional contributions are not precisely defined. The mass and width of the $f_{0}(1500)$ are compatible with earlier measurements, but less accurate. The parameters of the $3 \pi^{0}$ resonance with quantum numbers of the pion are $\mathrm{M}=1114 \mathrm{MeV}, \Gamma=340 \mathrm{MeV}$, if left free.

We would like to thank the technical staff of the LEAR machine group and of all the participating institutions for their invaluable contributions to the success of the experiment. We acknowledge financial support from the German Bundesministerium für Bildung, Wissenschaft, Forschung und Technologie, the Schweizerischer Nationalfonds, the British Particle Physics and Astronomy Research Council, the KFA Jülich, the U.S. Department of Energy and the National Science Research Fund Committee of Hungary (contract No. DE-FG03-87ER40323, DE-AC0376SF00098, DE-FG02-87ER40315 and OTKA F014357). N. Djaoshvili, F.-H. Heinsius and K.M. Crowe acknowledge support from the A. von Humboldt Foundation.

\section{References}

[1] Crystal Barrel Collaboration, I. Augustin et al., Nucl. Phys. A5 58 (1993) 3c

[2] Crystal Barrel Collaboration, V.V. Anisovich et al., Phys. Lett. B323 (1994) 233

[3] Crystal Barrel Collaboration, C. Amsler et al., Phys. Lett. B340 (1995) 259

[4] Crystal Barrel Collaboration, C. Amsler et. al., Phys. Lett. B342 (1995) 433

[5] Crystal Barrel Collaboration, C. Amsler et al., Phys. Lett. B353 (1995) 571

[6] Crystal Barrel Collaboration, C. Amsler et al., Phys. Lett. B355 (1995) 425

[7] V.V. Anisovich, D.V. Bugg, A.V. Sarantsev and B.S. Zou, Phys. Rev. D50 (1994) 1972

[8] V.V. Anisovich, D.V. Bugg, A.V. Sarantsev and B.S. Zou, Phys. Rev. D50 (1994) 4412

[9] V.V. Anisovich, A.A. Kondashov, Y.D. Prokoshkin, S.A. Sadovsky, A.V. Sarantsev, Phys. Lett. B355 (1995) 363

[10] S.U.Chung et al., Ann. d. Physik 4 (1995) 404

[11] G. Janssen, B.C. Pearce, K. Holinde and J. Speth, Phys. Rev. D52 (1995) 2690.
[12] B.S. Zou and D.V. Bugg, Phys. Rev. D50 (1994) 591

[13] D.V. Bugg et al., Phys. Lett. B353 (1995) 378

[14] A. Bettini et al., Nuovo Cimento A42 (1966) 695

[15] D. Bridges, I. Daftari and T.E. Kalogeropoulos, Phys. Rev. Lett. 57 (1986) 1535

[16] M. Gaspero, Nucl. Phys. A562 (1993) 407

[17] OBELIX Collaboration, A. Adamo et al., Nucl. Phys. A558 (1993) 13c

[18] Crystal Barrel Collaboration, C. Amsler et al., Phys. Lett. B322 (1994) 431

[19] Crystal Barrel Collaboration, E. Aker et al., Nucl. Instr. and Methods. A321 (1992) 69

[20] S.Resag, Ph. D. thesis, University of Bonn (1996)

[21] I. Scott, Ph. D. thesis, University of London (1996)

[22] L. Rosselet et al., Phys. Rev. D15 (1977) 574

[23] G. Grayer et al., Nucl. Phys. B75 (1974) 189

[24] M. Svec et al., Phys. Rev. D45 (1992) 55

[25] Particle Data Group (PDG), Phys. Rev. D50 (1994) 1479

[26] D.V. Bugg, A.V. Sarantsev and B.S. Zou, submitted to Nucl. Phys. B. 